\title{
SARS-CoV-2 infection in patients with primary central nervous system lymphoma
}

\author{
Alice Laurenge ${ }^{1} \cdot$ Renata Ursu $^{2}$. Caroline Houillier ${ }^{1} \cdot$ Basma Abdi $^{3,4} \cdot$ Gianpiero Tebano $^{5} \cdot$ Cyril Quemeneur $^{6}$. \\ Sylvain Choquet ${ }^{7} \cdot$ Roberta Di Blasi $^{8} \cdot$ Fernando Lozano ${ }^{1} \cdot$ Andrea Morales $^{1} \cdot$ Alberto Durán-Peña $^{1}$. \\ Lila Sirven-Villaros ${ }^{2} \cdot$ Bertrand Mathon $^{9} \cdot$ Karima Mokhtari $^{10} \cdot$ Franck Bielle $^{10} \cdot$ Nadine Martin-Duverneuil $^{11}$. \\ Jean-Yves Delattre ${ }^{1}$. Anne-Geneviève Marcelin ${ }^{3,4}$ • Valérie Pourcher ${ }^{4,5}$. Agusti Alentorn ${ }^{1} \cdot$ Ahmed Idbaih $^{1}$. \\ Antoine F. Carpentier ${ }^{2} \cdot$ Véronique Leblond $^{7} \cdot$ Khê Hoang-Xuan $^{1} \cdot$ Mehdi Touat $^{1}$
}

Received: 17 September 2020 / Revised: 26 October 2020 / Accepted: 8 November 2020 / Published online: 2 January 2021

(c) Springer-Verlag GmbH Germany, part of Springer Nature 2021

\begin{abstract}
Background Cancer patients may be at higher risk for severe coronavirus infectious disease-19 (COVID-19); however, the outcome of Primary Central Nervous System Lymphoma (PCNSL) patients with SARS-CoV-2 infection has not been described yet.

Methods We conducted a retrospective study within the Lymphomes Oculo-Cérébraux national network (LOC) to assess the clinical characteristics and outcome of SARS-CoV-2 infection in PCNSL patients (positive real-time polymerase chain reaction of nasopharyngeal swab or evocative lung computed tomography scan). We compared clinical characteristics between patients with severe (death and/or intensive care unit admission) and mild disease.

Results Between March and May 2020, 13 PCNSL patients were diagnosed with SARS-CoV-2 infection, 11 (85\%) of whom were undergoing chemotherapy at the time of infection. The mortality rate was $23 \%$ (3/13), and two additional patients (15\%) required mechanical ventilation. Two patients (15\%) had no COVID-19 symptoms. History of diabetes mellitus was more common in severe patients ( $3 / 5$ vs $0 / 8, p=0.03$ ). Two patients recovered from COVID-19 after mechanical ventilation during more than two weeks and resumed chemotherapy. In all, chemotherapy was resumed after COVID-19 recovery in nine patients (69\%) after a median delay of 16 days (range 3-32), none of whom developed unusual chemotherapy complication nor SARS-Cov2 reactivation.

Conclusion This preliminary analysis suggests that, while being at higher risk be for severe illness, PCNSL patients with COVID-19 might be treated maximally especially if they achieved oncological response at the time of SARS-CoV-2 infection. Chemotherapy might be resumed without prolonged delay in PCNSL patients with COVID-19.
\end{abstract}

Keywords COVID-19 $\cdot$ PCNSL

\section{Introduction}

Severe acute respiratory syndrome coronavirus-2 (SARS$\mathrm{CoV}-2$ ) is a new betacoronavirus responsible for the coronavirus infectious disease-19 (COVID-19) pandemic [1]. Models estimate that approximately $0.1-0.5 \%$ of infected patients

Electronic supplementary material The online version of this article (https://doi.org/10.1007/s00415-020-10311-w) contains supplementary material, which is available to authorized users.

Mehdi Touat

mehdi.touat@gmail.com

Extended author information available on the last page of the article die from COVID-19 [2, 3]. Risk factors for severe COVID19 in the general population include older age, male sex, and personal history of hypertension, obesity and diabetes mellitus [4]. In addition, emerging evidence suggest that cancer patients are at higher risk for severe COVID-19 (i.e., death, invasive ventilation, intensive care unit [ICU] admission) [5-8]. While several factors might explain this observation (e.g., comorbidities, poor health status, and systemic immunosuppression related to both the cancer and its treatment), the supporting evidence has been limited to studies pooling several cancer types [5-10]; therefore, limiting healthcare providers' ability to understand how COVID-19 severity 
may vary among cancer patients with various diagnosis, disease characteristics and treatment patterns.

Preliminary analyses aiming to analyze the outcome of SARS-CoV-2 infection in cancer patients suggest that patients with hematological malignancies may have a more severe COVID-19 [11-13]. Two recent reports indeed reported a higher case fatality rate for patients with hematologic malignancies when compared to patients with solid malignancies [6, 14]. However, to our knowledge, the outcome of Primary Central Nervous System Lymphoma (PCNSL) patients with SARS-CoV-2 infection has not been described yet. Given their intensive cancer treatment (antiCD20 therapy, corticosteroids, high-dose methotrexate) and underlying general and neurological condition, PCNSL patients may constitute a high-risk population for severe COVID-19. Although PCNSL are highly chemosensitive and potentially curable tumors, delays in treatment can be detrimental as PCNSL cells grow rapidly in the absence of treatment. Whether chemotherapy in PCNSL patients with SARS-CoV-2 infection can be suspended to mitigate the risks of both severe infection and cancer progression has not been addressed.

Here, we conducted a retrospective study in two expert centers from the Lymphomes Oculo-Cérébraux (LOC, dedicated to PCNSL care) national network in a high COVID-19 epidemic region to assess the clinical features and outcome of SARS-CoV-2 infection in PCNSL patients.

\section{Methods}

\section{Study design and participants}

We reviewed individual records of patients followed for PCNSL in two Parisian Hospitals from the national LOC network (La Pitié Salpêtrière and Saint Louis) and selected patients who had a SARS-CoV-2 infection between March 2020, and May 2020. Diagnosis of SARS-CoV-2 infection was based either on a positive real-time polymerase chain reaction (RT-PCR) of nasopharyngeal swab or on lung computed tomography (CT) scans (i.e., ground-glass opacities, multilobe or posterior involvement, bilateral pneumonia distribution $[15,16])$. In both hospitals, all patients with PCNSL had systematic screening for SARS-CoV-2 infection with either a RT-PCR screening or a lung CT-scan at the time of admission, regardless of whether they had COVID19 symptoms. The study was conducted in compliance with the Declaration of Helsinki. Approval was obtained from the local ethics committee (IRB approval 2020CER-202028).

\section{Procedures}

Epidemiological, clinical and laboratory data, radiology reports, therapy details and outcomes were obtained retrospectively from electronic medical records. Oncological responses were defined by the International PCNSL Collaborative Group (IPCG) criteria [17]. For RT-PCR, ORF and gene $\mathrm{E}$ regions were analyzed; the end result was considered positive if one or the other was positive. The presence of anti-SARS-CoV-2 IgG in the serum was assessed using a chemiluminescent microparticle immunoassay (ARCHITECT i System, Abbott).

\section{Statistical analysis}

Continuous variables were expressed as median for abnormally distributed data. Categorical variables were expressed as frequency rates and percentages. Mean were compared using independent group Wilcoxon-Mann-Whitney tests. Proportions for categorical variables were compared using Fischer tests. All statistical analyses were performed using the R software, version 3.6.3 (http://www.r-project.org/). All tests were two-sided and $P$ values of less than 0.05 were regarded as statistically significant. Analyses were not adjusted for multiple comparisons. Findings should be interpreted as exploratory and descriptive.

\section{Results}

\section{Patient characteristics}

We identified 13 PCNSL patients with confirmed SARSCoV-2 infection between March and May 2020. Patients' characteristics at baseline are provided in Table 1. Seven patients were admitted for their PCNSL treatment before SARS-CoV-2 infection first symptoms or diagnosis, while six patients were admitted for the management of COVID19. The median age was 65 years (range 35-83) and $62 \%$ were females. The median delay between PCNSL diagnosis and SARS-CoV-2 infection was 3.7 months (range 1-165 months). Three patients were receiving first-line treatment without response evaluation yet available, six patients had a complete response (CR), two had a partial response (PR), and the remaining two patients had a relapsed or refractory disease. At the time of SARS-CoV-2 infection first symptoms or diagnosis, the majority of the patients (11/13) were undergoing chemotherapy, 7/11 of whom were receiving R-MPV (rituximab, high-dose methorexate, procarbazine, vincristine and methylprednisone) poly-chemotherapy (four CR, one PR, and two not yet evaluated). Two 
Table 1 Clinical characteristics of patients with PCNSL and COVID-19

\begin{tabular}{|c|c|c|c|}
\hline Variable & $\begin{array}{l}\text { Total } \\
n=13\end{array}$ & $\begin{array}{l}\text { Mild } \\
n=8(61.5 \%)\end{array}$ & $\begin{array}{l}\text { Severe } \\
n=5(38.5 \%)\end{array}$ \\
\hline Male gender-no. $(\%)$ & $5(38.5)$ & $2(25)$ & $3(60)$ \\
\hline \multicolumn{4}{|l|}{ Age, years-no. $(\%)$} \\
\hline $18-60$ & $4(30.8)$ & $3(37.5)$ & $1(20)$ \\
\hline $60-80$ & $8(61.5)$ & $4(50)$ & $4(80)$ \\
\hline$>80$ & $1(7.7)$ & $1(12.5)$ & $0(0)$ \\
\hline \multicolumn{4}{|l|}{ Underlying condition-no. (\%) } \\
\hline Obesity $\left(\mathrm{BMI} \geq 30 \mathrm{~kg} / \mathrm{m}^{2}\right)^{\dagger}$ & $4(30.8)$ & $2(25)$ & $2(50)$ \\
\hline Overweight or Obesity $\left(\mathrm{BMI} \geq 25 \mathrm{~kg} / \mathrm{m}^{2}\right)^{\dagger}$ & $9(69.2)$ & $5(62.5)$ & $4(100)$ \\
\hline Diabetes & $3(23.1)$ & $0(0)^{*}$ & $3(60)^{*}$ \\
\hline High blood pressure & $7(53.8)$ & $5(62.5)$ & $2(40)$ \\
\hline Delay since initial lymphoma diagnosis, months-median (range) & $3.7(1.0-164.9)$ & $4(1.6-55.6)$ & $3.1(1.0-164.9)$ \\
\hline \multicolumn{4}{|l|}{ KPS at baseline-no. (\%) } \\
\hline $40-50$ & $5(38.5)$ & $3(37.5)$ & $2(40)$ \\
\hline $60-70$ & $4(30.8)$ & $3(37.5)$ & $1(20)$ \\
\hline $80-90$ & $4(30.8)$ & $2(25)$ & $2(40)$ \\
\hline Steroids intake at baseline - no. $(\%)^{\dagger \dagger}$ & $3(23.1)$ & $1(12.5)$ & $2(40)$ \\
\hline Chemotherapy at baseline-no. $(\%)^{\#}$ & $11(84.6)$ & $7(87.5)$ & $4(80)$ \\
\hline Anti-CD20 therapy at baseline-no. (\%) & $9(69.2)$ & $6(75)$ & $3(60)$ \\
\hline Chemotherapy line - median (range) & $1(1-5)$ & $1(1-2)$ & $1(1-5)$ \\
\hline Remission without treatment-no. $(\%)$ & $1(7.7)$ & $1(12.5)$ & $0(0)$ \\
\hline First line-no. $(\%)$ & $10(76.9)$ & $7(87.5)$ & $3(60)$ \\
\hline Fifth line-no. $(\%)$ & $1(7.7)$ & 0 & $1(20)$ \\
\hline Chemotherapy to resume (relapse)-no. $(\%)$ & $1(7.7)$ & 0 & $1(20)$ \\
\hline \multicolumn{4}{|l|}{ Hematologic malignancy status-no. (\%) } \\
\hline Initial diagnosis or first-line treatment without evaluation & $3(23.1)$ & $2(25)$ & $1(20)$ \\
\hline Relapsed or refractory & $2(15.4)$ & $0(0)$ & $2(40)$ \\
\hline Partial response & $2(15.4)$ & $1(12.5)$ & $1(20)$ \\
\hline Complete response & $6(46.2)$ & $5(62.5)$ & $1(20)$ \\
\hline
\end{tabular}

$\dagger$ Data missing for one patient

$\dagger \geq 10 \mathrm{mg}$ prednisone-equivalent

\#One patient was treated with revlimid

$* p<0.05$ for mild vs severe patients (Mann-Whitney or Fisher test)

additional patients were undergoing anti-CD20 therapy concomitant with high-dose methotrexate (one PR and one not yet evaluated). In total, nine patients were undergoing anti-CD20 therapy. The two remaining patients were undergoing, respectively, high-dose Methotrexate concomitant with Temozolomide (achieved CR) and lenalidomide (for relapse).

\section{Symptoms, diagnosis and treatment of COVID-19}

Patients' COVID-19 symptoms at baseline are provided in Supplementary Table 1. The most common symptom was fever (10 patients). In addition, six patients had cough and six had shortness of breath. Three patients presented with confusion or clear increase in pre-existing confusion.
No other neurological symptom was reported. Two patients without COVID-19 symptoms were diagnosed following systematic RT-PCR screening for SARS-CoV-2 at the time of their admission for chemotherapy. All but three patients had an initial positive RT-PCR of nasopharyngeal swab. Two of the remaining patients had a positive RT-PCR after a first negative test, and the last patient had acute respiratory failure with a lung CT-scan showing pulmonary abnormalities consistent with COVID-19 (bilateral ground-glass opacities).

As regard COVID-19 treatment, four patients were treated with hydroxychloroquine (chloroquine derivative), one with anakinra (interleukin 1 antagonist) and one with remdesivir (RNA polymerase inhibitor), according to local guidelines. In addition, three patients underwent 
corticosteroid therapy (prednisone $1 \mathrm{mg} / \mathrm{kg}$ for 7-14 days) because of radiological evidence of pulmonary fibrosis on follow-up lung CT-scans. All patients received antithrombotic therapy (either prophylaxis dose, intermediate or therapeutic dose) and none was diagnosed with venous thromboembolism during the course of COVID-19. The number of patients was too small to compare outcomes according to pattern of COVID-19 treatment.

Table 2 COVID-19 severity in patients with PCNSL and COVID-19

\begin{tabular}{ll}
\hline Severity-no. $(\%)$ & $n=13$ \\
\hline Asymptomatic & $2(15.4)$ \\
Symptomatic, requiring $\leq 3 \mathrm{~L} / \mathrm{min}$ oxygen & $4(30.8)$ \\
Symptomatic, requiring $>3 \mathrm{~L} / \mathrm{min}$ oxygen & $2(15.4)$ \\
Requiring mechanical ventilation & $2(15.4)$ \\
Death & $3(23.1)$ \\
\hline
\end{tabular}

\section{Outcome of SARS-CoV-2 infection}

At last follow-up (June 2020), 10 patients (77\%) were still alive (see Table 2). The median follow-up was 64 days (range 4-94), 71 days for patients who remained alive (range 33-94), and 4-12 days for the three deceased patients. These three patients, considered ineligible for ICU admission (poor health status, $n=2$; refractory PCNSL, $n=2$ ), died shortly following their COVID-19 diagnosis. Two patients required ICU admission for the management of respiratory failure (see Table 2). Nine patients (69\%) required oxygen for a median time of 12 days (range 1-73 days) (Fig. 1). Patients were symptomatic for a median duration of 13 days (range 4-88). Finally, two patients developed no COVID-19 symptom, despite having prolonged RT-PCR positivity (46 and 47 days vs $22-28$ days for the remaining patients).

Among the two patients who required ICU admission, both had extended lesions on the initial lung CT-scan and required mechanical ventilation (one non-invasive for 22 days; one invasive for 48 days) (Fig. 2). Interestingly, both patients achieved good respiratory and general

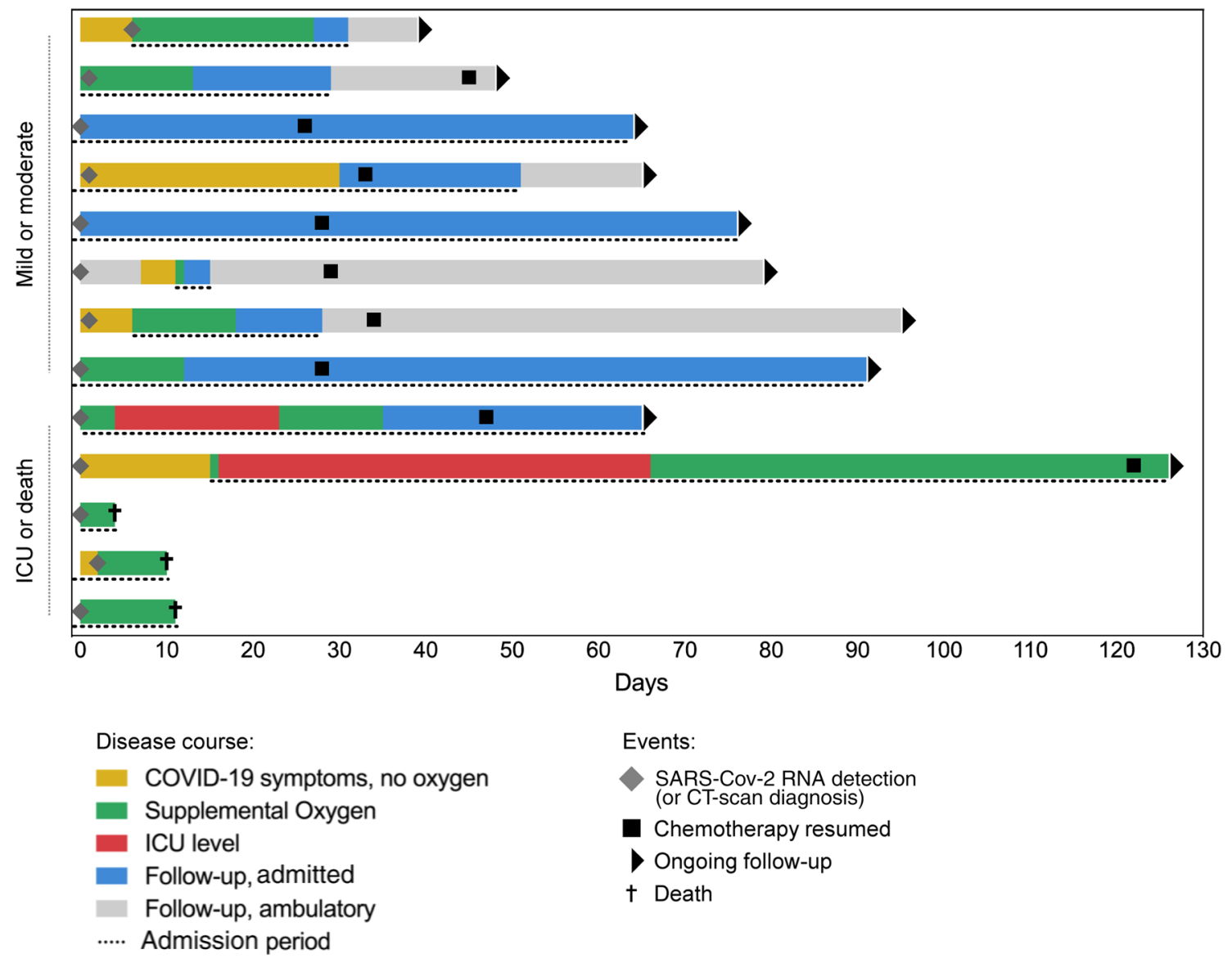

Fig. 1 Timeline of illness onset, SARS-CoV-2 RNA detection, admission, oxygen therapy, symptom resolution and chemotherapy resumption among the cohort 
COVID-19 outcome and were discharged from the ICU after 23 and 66 days, respectively.

\section{Outcome of PCNSL and evolution after chemotherapy resumption}

According to local guidelines, chemotherapy was suspended immediately after SARS-CoV-2 infection diagnosis in all patients under treatment $(n=11)$, for a median delay of 32 days (range 26-122). During the follow-up period, chemotherapy was resumed in nine patients $(69 \%$, all alive patients who were under chemotherapy at baseline). It was postponed for 2-4 weeks in five patients and for more than four weeks in four patients. The median time between the last COVID-19 symptom and chemotherapy resumption was 16 days for the seven symptomatic patients (range 3-32 days) (Fig. 1).

Control RT-PCR were performed before chemotherapy resumption in six patients, of whom two patients (33\%) remained positive. Both patients nonetheless resumed their chemotherapy without evidence of SARS-Cov2 reactivation or other related complication. A negative control RT-PCR was further obtained for both patients three weeks after chemotherapy was resumed. Blood serology was performed before chemotherapy resumption in six patients, and only two patients (33\%) had a significant IgG rate at the time of chemotherapy resumption (median time after SARS-CoV-2 infection: 30 days [range 26-33]). No systematic CT-scan was performed before chemotherapy resumption.

No unexpected clinical nor biological evolution was observed after chemotherapy was resumed (Supplementary Fig. 1). Of note, both patients admitted in the ICU were also able to resume their induction chemotherapy regimen with high-dose methotrexate. Further evaluation confirmed complete oncological response in both patients (Fig. 2).

\section{Final outcome and prognostic factors}

At last follow-up 10/13 patients were alive. Three died shortly following COVID-19 diagnosis, without being admitted to ICU due to adverse prognostic factors at the time of COVID-19 (see above); none died directly from PCNSL progression. All ten alive patients recovered good respiratory function (no oxygen required, no shortness of breath). One patient developed PCNSL progression during the follow-up period. This patient was receiving $1^{\text {st }}$ line chemotherapy, had a progressive deterioration of general health status with a KPS of 50\%, and achieved only partial response at the time of SARS-CoV-2 infection. She remained asymptomatic for SARS-CoV-2 infection and was able to resume chemotherapy but worsened progressively due to PCNSL progression.
To better understand prognostic factors of COVID-19 in PCNSL patients, we performed an exploratory analysis comparing clinical characteristics between patients with severe (death and/or ICU transfer, 5 patients, 38\%) and mild disease (8 patients, 62\%) (Tables 1, 2, and supplementary Table 1). History of diabetes was less common in mild patients as compared to severe patients $(0 / 8$ vs. $3 / 5$, $p=0.03$, Supplementary Table 1 ). The other clinical data at baseline did not differ significantly between mild and severe patients. Patients with severe COVID-19 outcome tended to have received more than one line of chemotherapy for PCNSL (severe disease in 3/10 patients who received only one line of chemotherapy vs $2 / 3$ patients who received more than one line). We found no association between the administration of rituximab (anti-CD20) and severity (severe disease in $3 / 9$ patients who received rituximab vs $1 / 2$ patients who underwent chemotherapy without rituximab). Patients with severe COVID-19 had a significantly higher neutrophil count at baseline (median $4460 \mathrm{vs} 1695 / \mathrm{mm}^{3}, p<0.05$ ). Other biological data at baseline did not significantly differ according to the prognosis (Supplementary Table 2).

\section{Discussion}

To our knowledge, this is the first report of PCNSL patients with SARS-CoV-2 infection. We describe the severity of the infection and prognostic factors of COVID-19 in PCNSL.

Understanding the factors associated with severe COVID19 is an important public health issue. Our analysis suggests that PCNSL patients might be at higher risk be for severe COVID-19, with $38.5 \%$ severe forms and $23 \%$ fatality rate in our PCNSL series vs. 6.1-21.4\% ICU admission and 7.5\% fatality rate reported in the general population [18-20]. The prognosis of SARS-CoV-2 infection in PCNSL patients in our series was comparable with the one reported in patients with solid malignancies, with approximately $25 \%$ mortality rate $[6,14]$, and slightly better than patients with other hematological malignancies (37-61.5\% mortality rate) $[6,11,14,21]$. Of note, our high fatality rate could be explained, at least in part, by limitations of care due to poor prognosis or health status leading to less intensive COVID19 treatment. Our patients also had a higher median age and more history of diabetes mellitus-two known risk factors for severe disease $[22,23]$ - than the general population of reported COVID-19 patients, which could in part explain their worse prognosis.

While PCNSL patients are at high-risk for severe COVID19 , the asymptomatic rate of SARS-CoV-2 infection (15\% in our series) was identical to that of the general population [24-30]. Our patients had more frequent shortness of breath as reported in the general population: $46 \%$ in our series vs $18.7 \%$ [18], which was in line with our observation of more 


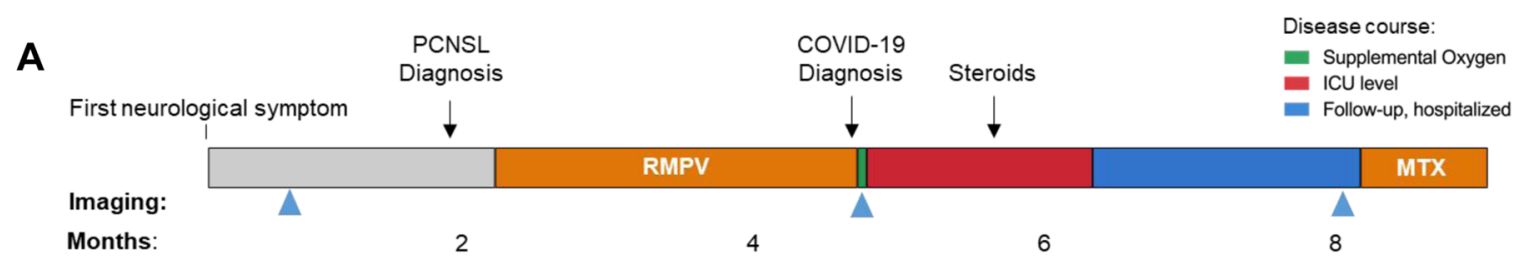

Baseline

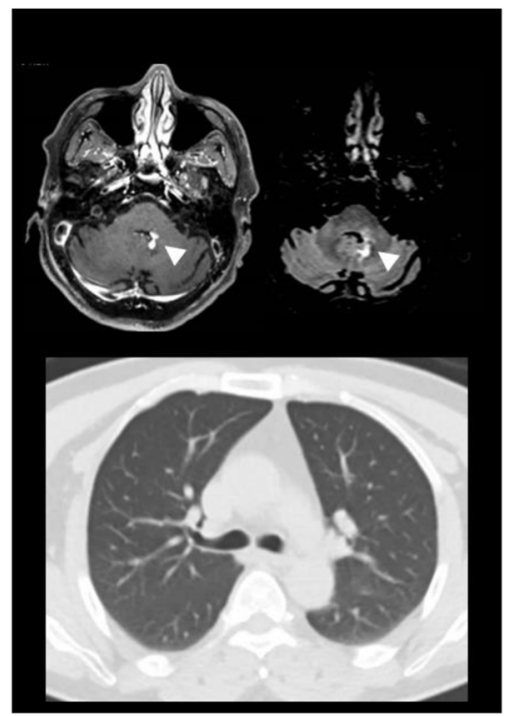

B

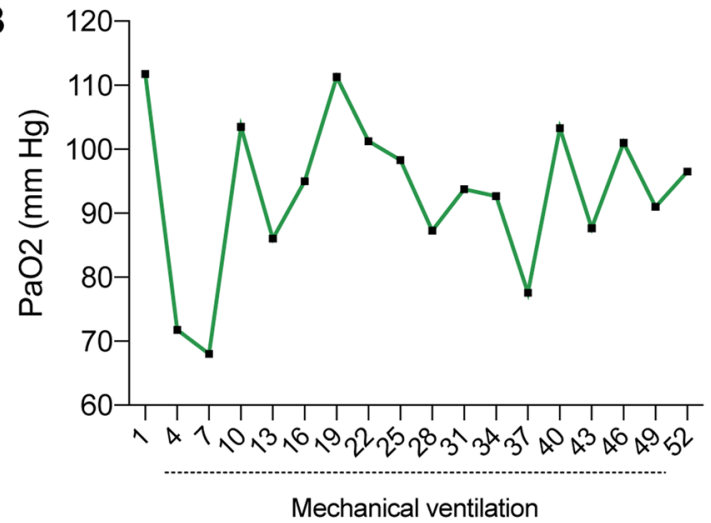

D

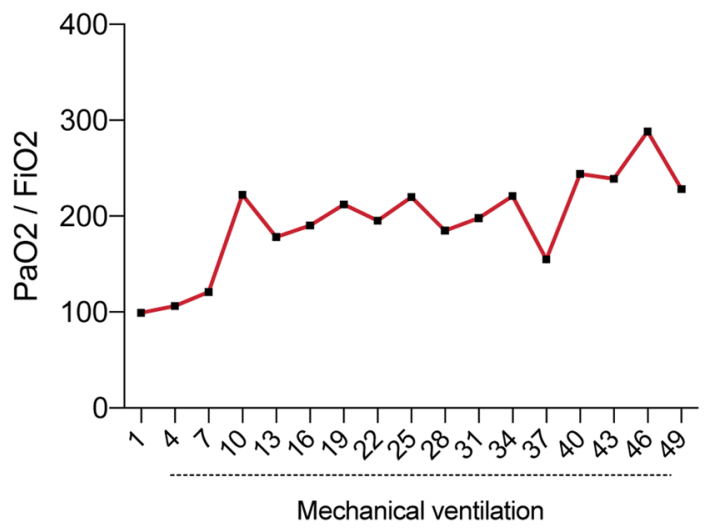

SARS-Cov2 Infection

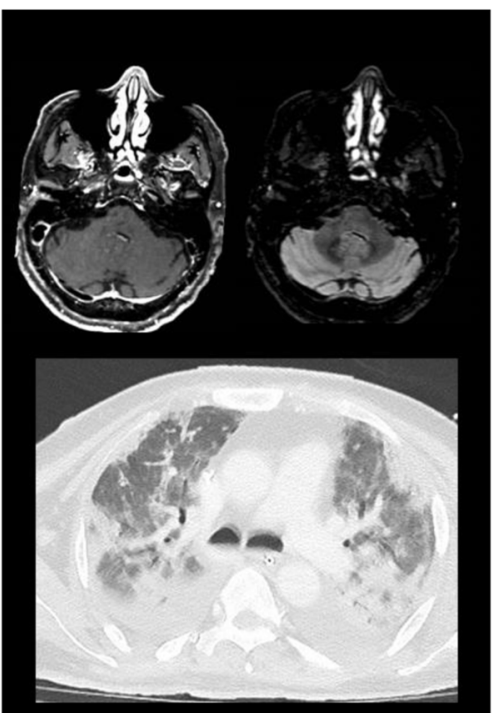

C



E




4Fig. 2 Severe COVID-19 Infection in a 65-year-old patient with PCNSL. a Clinical Timeline. Top, timeline; bottom, brain MRI (left, T1-Gad; right, FLAIR) and lung CT-scan images during initial PCNSL diagnosis (left), SARS-Cov2 infection, and the recovery phase. b-e Evolution of gas exchange parameters during SARS-Cov2 infection

severe COVID-19 in the PCNSL population. He and al. also reported more frequent dyspnea in hematological patients as compared to the general population (77\% vs $27 \%$ ) [11]. All patients who underwent chemotherapy at baseline were regularly admitted for treatment, which constitutes a risk factor for SARS-CoV-2 infection.

Our study has several limitations related to its retrospective nature, small sample size due to the low prevalence of PCNSL, and confounding co-variates such as chemotherapy and heterogeneous disease stage. Despite these limitations, our study provides preliminary data which will help clinical decisions regarding the management of COVID-19 in this rare patient population. PCNSL patients who do not receive chemotherapy have a rapidly fatal course. Although current guidelines suggest suspending chemotherapy in cancer patients with COVID-19, the modality for treatment resumption and adjustment are poorly defined. In our series, chemotherapy was resumed after a median delay of 16 days in all patients after COVID-19 clinical recovery, regardless of control SARS-CoV-2 RT-PCR or serology results. We observed no unexpected evolution after chemotherapy resumption, with a median follow-up of 1.5 months.

We report two patients with severe COVID-19 (mechanical ventilation more than 2 weeks) who were both able to resume PCNSL chemotherapy without experiencing disease progression. According to current consensus guidelines [31], decision of ICU transfer and intensity of treatment in cancer patients with COVID-19 should be adjusted depending on the status of the viral outbreak (e.g., current R0 index, basic reproduction number), availability of hospital technical and human resources (e.g., ICU ventilation capacities), as well as risk factors for adverse outcomes. Our data suggests that PCNSL patients might be treated intensively especially if they have reasonable life expectancy (e.g., patients with good performance status, who are in first-line chemotherapy and patients who already achieved a complete or partial response).

In conclusion, our preliminary data suggest that, while being at higher risk for severe illness, PCNSL patients with COVID-19 might be treated intensively especially if they have reasonable life expectancy. No evidence of SARS-Cov2 reactivation was found in PCNSL patients who resumed chemotherapy after COVID-19 clinical recovery. This preliminary data will inform guidelines for the management of PCNSL and cancer patients in the context of COVID19 and future outbreaks [32-36]. Future studies should address which PCNSL patients have the highest risk for severe COVID-19, the long-term outcomes of COVID-19 in PCNSL patients, and how chemotherapy should be optimized in this population to mitigate the risks of both severe infection and cancer progression.

Acknowledgements The authors would like to thank the patients who took part in the study and their families, as well as the staff, research coordinators, and investigators at each participating institution. We gratefully acknowledge the support of the CoCo Neurosciences study group: Steering Committee (Pitié-Salpêtrière Hospital, Paris): Cecile Delorme, Jean-Christophe Corvol, Jean-Yves Delattre, Stephanie Carvalho, Sandrine Sagnes. Scientific Committee (Pitié-Salpêtrière Hospital, Paris): Bruno Dubois, Vincent Navarro, Celine Louapre, Tanya Stojkovic, Ahmed Idbaih, Charlotte Rosso, David Grabli, Ana Zenovia Gales, Bruno Millet, Benjamin Rohaut, Eleonore Bayen, Sophie Dupont, Gaelle Bruneteau, Stephane Lehericy, Danielle Seilhean, Alexandra Durr, Foudil Lamari, Marion Houot, Vanessa Batista Brochard. Principal investigators: Pitié-Salpêtrière Hospital (Paris): Sophie Dupont, Catherine Lubetzki, Danielle Seilhean, Pascale Pradat-Diehl, Charlotte Rosso, Khe Hoang-Xuan, Bertrand Fontaine, Lionel Naccache, Philippe Fossati, Isabelle Arnulf, Alexandra Durr, Alexandre Carpentier, Stephane Lehericy, Yves Edel; Rothschild Hospital (Paris): Gilberte Robain, Philippe Thoumie; Avicenne Hospital (Bobigny): Bertrand Degos; Sainte-Anne Hospital (Paris): Tarek Sharshar; SaintAntoine Hospital (Paris): Sonia Alamowitch, Emmanuelle ApartisBourdieu, Charles-Siegried Peretti; Saint-Louis Hospital (Paris): Renata Ursu; Tenon Hospital (Paris): Nathalie Dzierzynski; Charles Foix Hospital (Ivry): Kiyoka Kinugawa Bourron, Joel Belmin, Bruno Oquendo, Eric Pautas, Marc Verny. Co-investigators: Pitié-Salpêtrière Hospital (Paris): Cecile Delorme, Jean-Christophe Corvol, Jean-Yves Delattre, Yves Samson, Sara Leder, Anne Leger, Sandrine Deltour, Flore Baronnet, Ana Zenovia Gales,Stephanie Bombois, Mehdi Touat, Ahmed Idbaih, Marc Sanson, Caroline Dehais, Caroline Houillier, Florence Laigle-Donadey, Dimitri Psimaras, Agusti Alenton, Nadia Younan, Nicolas Villain, David Grabli, Maria del Mar Amador, Gaelle Bruneteau, Celine Louapre, Louise-Laure Mariani, Nicolas Mezouar, Graziella Mangone, Aurelie Meneret, Andreas Hartmann, Clement Tarrano, David Bendetowicz, Pierre-François Pradat, Michel Baulac, Sara Sambin, Phintip Pichit, Florence Chochon, Adele Hesters, Bastien HerlinAn Hung Nguyen, Valerie Procher, Alexandre Demoule, Elise Morawiec, Julien Mayaux, Morgan Faure, Claire Ewenczyk, Giulia Coarelli, Anna Heinzmann, Tanya Stojkovic, Marion Masingue, Guillaume Bassez, Vincent Navarro, Isabelle An, Yulia Worbe, Virginie Lambrecq, Rabab Debs, Esteban Munoz Musat, Timothee Lenglet, Virginie Lambrecq, Aurelie Hanin, Lydia Chougar, Nathalia Shor, Nadya Pyatigorskaya, Damien Galanaud, Delphine Leclercq, Sophie Demeret, Benjamin Rohaut, Albert Cao, Clemence Marois, Nicolas Weiss, Salimata Gassama, Loic Le Guennec, Vincent Degos, Alice Jacquens, Thomas Similowski, Capucine Morelot-Panzini, Jean-Yves Rotge, Bertrand Saudreau, Bruno Millet, Victor Pitron, Nassim Sarni, Nathalie Girault, Redwan Maatoug, Ana Zenovia Gales, Smaranda Leu, Eleonore Bayen, Lionel Thivard, Karima Mokhtari, Isabelle Plu; Sainte-Anne Hospital (Paris): Bruno Gonçalves; Saint-Antoine Hospital (Paris): Laure Bottin, Marion Yger; Rothschild Hospital (Paris): Gaelle Ouvrard, Rebecca Haddad; Charles Foix Hospital (Ivry): Flora Ketz, Carmelo Lafuente, Christel Oasi. Other Contributors: Associated centers (Lariboisière Hospital, Paris): Bruno Megabarne, Dominique Herve; Clinical Research Associates (ICM, Pitié-Salpêtrière Hospital, Paris): Haysam Salman, Armelle Rametti-Lacroux, Alize Chalançon, Anais Herve, Hugo Royer, Florence Beauzor, Valentine Maheo, Christelle Laganot, Camille Minelli, Aurelie Fekete, Abel Grine, Marie Biet, Rania Hilab, Aurore Besnard, Meriem Bouguerra, Gwen Goudard, Saida Houairi, Saba Al-Youssef, Christine Pires, Anissa Oukhedouma, 
Katarzyna Siuda-Krzywicka, Tal Seidel Malkinson; (Saint-Louis Hospital, Paris): Hanane Agguini; Data Manager (ICM, Paris): Safia Said; Statistician (ICM, Paris): Marion Houot.

Author contributions AL, KHX and MT designed the study. AL, RU, $\mathrm{CQ}, \mathrm{SC}$, and MT collected the data. AL and MT reviewed and analyzed the clinical data. AL and MT wrote the initial draft, with input from all authors. All authors participated in data analysis, reviewed and approved the final manuscript.

Funding This work was in part supported by a grant INCa-DGOSInserm_12560 of the SiRIC CURAMUS, Institut National du Cancer (INCa). The Cohort COVID-19 Neurosciences (CoCo Neurosciences) study was sponsored by APHP and funded by the generous support of the FIA (Fédération Internationale pour l'Automobile) Foundation and donors of the Paris Brain Institute - ICM. The research leading to these results has received funding from the program "Investissements d'avenir" ANR-10- IAIHU-06.

\section{Compliance with ethical standards}

Conflicts of interest MT reports consulting or advisory role from Agios Pharmaceutical, Integragen, and Taiho Oncology, outside the submitted work; travel, accommodations, expenses from Merck Sharp $\&$ Dome, outside the submitted work. All authors report no disclosures relevant to the manuscript.

Ethical approval The study was conducted in compliance with the Declaration of Helsinki. Approval was obtained from the local ethics committee (IRB approval 2020CER-202028).

Consent to participate All patients gave their informed consent prior to their inclusion in the study.

Consent for publication All authors agreed with the content of the manuscript and all gave explicit consent to submit.

\section{References}

1. Wu F et al (2020) A new coronavirus associated with human respiratory disease in China. Nature 579(7798):265-269. https://doi. org/10.1038/s41586-020-2008-3

2. Salje $\mathrm{H}$ et al (2020) Estimating the burden of SARS-CoV-2 in France. Science 369(6500):208-211. https://doi.org/10.1126/scien ce.abc 3517

3. Liang L-L, Tseng C-H, Ho HJ, Wu C-Y (2020) Covid-19 mortality is negatively associated with test number and government effectiveness. Sci Rep 10(1):12567. https://doi.org/10.1038/s4159 8-020-68862-x

4. Zhou F et al (2020) Clinical course and risk factors for mortality of adult inpatients with COVID-19 in Wuhan, China: a retrospective cohort study. Lancet Lond Engl 395(10229):1054-1062. https ://doi.org/10.1016/S0140-6736(20)30566-3

5. Liang $\mathrm{W}$ et al (2020) Cancer patients in SARS-CoV-2 infection: a nationwide analysis in China. Lancet Oncol 21(3):335-337. https ://doi.org/10.1016/S1470-2045(20)30096-6

6. Mehta V et al (2020) Case fatality rate of cancer patients with COVID-19 in a New York hospital system. Cancer Discov 10(7):935-941. https://doi.org/10.1158/2159-8290.CD-20-0516

7. Dai M et al (2020) Patients with cancer appear more vulnerable to SARS-CoV-2: a multicenter study during the COVID-19 outbreak.
Cancer Discov 10(6):783-791. https://doi.org/10.1158/21598290.CD-20-0422

8. Xia Y, Jin R, Zhao J, Li W, Shen H (2020) Risk of COVID-19 for patients with cancer. Lancet Oncol 21(4):e180. https://doi. org/10.1016/S1470-2045(20)30150-9

9. Zhang L et al (2020) Clinical characteristics of COVID-19-infected cancer patients: a retrospective case study in three hospitals within Wuhan, China. Ann Oncol Off J Eur Soc Med Oncol 31(7):894-901. https://doi.org/10.1016/j.annonc.2020.03.296

10. Wang $J$ et al (2020) Systematic investigations of COVID-19 in 283 cancer patients. medRxiv. https://doi. org/10.1101/2020.04.28.20083246

11. He W et al (2020) COVID-19 in persons with haematological cancers. Leukemia 34(6):1637-1645. https://doi.org/10.1038/s4137 5-020-0836-7

12. Martín-Moro F et al (2020) Survival study of hospitalised patients with concurrent COVID-19 and haematological malignancies. $\mathrm{Br}$ J Haematol. https://doi.org/10.1111/bjh.16801

13. Passamonti $\mathrm{F}$ et al (2020) Clinical characteristics and risk factors associated with COVID-19 severity in patients with haematological malignancies in Italy: a retrospective, multicentre, cohort study. Lancet Haematol. https://doi.org/10.1016/S2352 $-3026(20) 30251-9$

14. Meng Y et al (2020) Cancer history is an independent risk factor for mortality in hospitalized COVID-19 patients: a propensity score-matched analysis. J Hematol Oncol 13(1):75. https://doi. org/10.1186/s13045-020-00907-0

15. Caruso D et al (2020) Chest CT Features of COVID-19 in Rome, Italy. Radiology 296(2):E79-E85. https://doi.org/10.1148/radio 1.2020201237

16. Salehi S, Abedi A, Balakrishnan S, Gholamrezanezhad A (2020) Coronavirus disease 2019 (COVID-19): a systematic review of imaging findings in 919 patients. AJR Am J Roentgenol 215(1):87-93. https://doi.org/10.2214/AJR.20.23034

17. Abrey LE et al (2005) Report of an international workshop to standardize baseline evaluation and response criteria for primary CNS lymphoma. J Clin Oncol Off J Am Soc Clin Oncol 23(22):5034-5043. https://doi.org/10.1200/JCO.2005.13.524

18. Guan W-J et al (2020) Clinical characteristics of coronavirus disease 2019 in China. N Engl J Med 382(18):1708-1720. https:// doi.org/10.1056/NEJMoa2002032

19. Chen J et al (2020) Clinical progression of patients with COVID-19 in Shanghai, China. J Infect 80(5):e1-e6. https://doi. org/10.1016/j.jinf.2020.03.004

20. Immovilli P, Morelli N, Antonucci E, Radaelli G, Barbera M, Guidetti D (2020) COVID-19 mortality and ICU admission: the Italian experience. Crit Care Lond Engl 24(1):228. https://doi. org/10.1186/s13054-020-02957-9

21. Lee LYW et al (2020) COVID-19 prevalence and mortality in patients with cancer and the effect of primary tumour subtype and patient demographics: a prospective cohort study. Lancet Oncol. https://doi.org/10.1016/S1470-2045(20)30442-3

22. Huang I, Lim MA, Pranata R (2020) Diabetes mellitus is associated with increased mortality and severity of disease in COVID19 pneumonia - a systematic review, meta-analysis, and metaregression. Diabetes Metab. Syndr 14(4):395-403. https://doi. org/10.1016/j.dsx.2020.04.018

23. Roncon L, Zuin M, Rigatelli G, Zuliani G (2020) Diabetic patients with COVID-19 infection are at higher risk of ICU admission and poor short-term outcome. J Clin Virol Off Publ Pan Am Soc Clin Virol 127:104354. https://doi.org/10.1016/j.jcv.2020.104354

24. Nishiura $\mathrm{H}$ et al (2020) Estimation of the asymptomatic ratio of novel coronavirus infections (COVID-19). Int J Infect Dis 94:154 155. https://doi.org/10.1016/j.ijid.2020.03.020

25. Mizumoto K, Kagaya K, Zarebski A, Chowell G (2020) Estimating the asymptomatic proportion of coronavirus disease 2019 
(COVID-19) cases on board the Diamond Princess cruise ship, Yokohama, Japan, 2020. Eurosurveillance 25(10):2000180. https ://doi.org/10.2807/1560-7917.ES.2020.25.10.2000180

26. $\mathrm{Ng} \mathrm{O}-\mathrm{T}$ et al (2020) SARS-CoV-2 infection among travelers returning from Wuhan, China. N Engl J Med 382(15):1476-1478. https://doi.org/10.1056/NEJMc2003100

27. Park SY et al (2020) Coronavirus disease outbreak in call center, South Korea. Emerg Infect Dis 26(8):1666-1670. https://doi. org/10.3201/eid2608.201274

28. Wiersinga WJ, Rhodes A, Cheng AC, Peacock SJ, Prescott HC (2020) Pathophysiology, transmission, diagnosis, and treatment of coronavirus disease 2019 (COVID-19): A Review. JAMA. https ://doi.org/10.1001/jama.2020.12839

29. Byambasuren O, Cardona M, Bell K, Clark J, McLaws M-L, Glasziou P (2020) Estimating the extent of asymptomatic COVID19 and its potential for community transmission: systematic review and meta-analysis. Infect Dis (except HIV/AIDS). https:// doi.org/10.1101/2020.05.10.20097543

30. Tabata $\mathrm{S}$ et al (2020) Clinical characteristics of COVID-19 in 104 people with SARS-CoV-2 infection on the diamond princess cruise ship: a retrospective analysis. Lancet Infect Dis. https://doi. org/10.1016/S1473-3099(20)30482-5

31. Curigliano $\mathrm{G}$ et al (2020) Managing cancer patients during the COVID-19 pandemic: an ESMO interdisciplinary expert consensus. Ann Oncol Off J Eur Soc Med Oncol. https://doi. org/10.1016/j.annonc.2020.07.010

32. NCCN, NCCN COVID-19 Resources. https://www.ncen.org/covid $-19 /$ (consulté le juin 08, 2020).

33. ASCO, ASCO Coronavirus Resources, ASCO, mars 06, 2020. https://www.asco.org/asco-coronavirus-information (consulté le juin 08, 2020).

34. ESMO. ESMO COVID-19 and Cancer. https://www.esmo.org/ covid-19-and-cancer (consulté le juin 08, 2020).

35. Kutikov A, Weinberg DS, Edelman MJ, Horwitz EM, Uzzo RG, Fisher RI (2020) A war on two fronts: cancer care in the time of COVID-19. Ann Intern Med 172(11):756-758. https://doi. org/10.7326/M20-1133

36. Gosain R, Abdou Y, Singh A, Rana N, Puzanov I, Ernstoff MS (2020) COVID-19 and cancer: a comprehensive review. Curr Oncol Rep 22(5):53. https://doi.org/10.1007/s11912-020-00934-7

Publisher's Note Springer Nature remains neutral with regard to jurisdictional claims in published maps and institutional affiliations.

\section{Affiliations}

\section{Alice Laurenge ${ }^{1} \cdot$ Renata Ursu $^{2}$. Caroline Houillier ${ }^{1}$ - Basma Abdi ${ }^{3,4}$. Gianpiero Tebano ${ }^{5}$. Cyril Quemeneur ${ }^{6}$ Sylvain Choquet ${ }^{7} \cdot$ Roberta Di Blasi $^{8} \cdot$ Fernando Lozano ${ }^{1} \cdot$ Andrea Morales $^{1}$. Alberto Durán-Peña ${ }^{1}$. Lila Sirven-Villaros ${ }^{2} \cdot$ Bertrand Mathon $^{9} \cdot$ Karima Mokhtari $^{10}$. Franck Bielle $^{10} \cdot$ Nadine Martin-Duverneuil $^{11}$. Jean-Yves Delattre ${ }^{1}$. Anne-Geneviève Marcelin ${ }^{3,4}$. Valérie Pourcher ${ }^{4,5}$. Agusti Alentorn ${ }^{1} \cdot$ Ahmed Idbaih $^{1}$. Antoine F. Carpentier ${ }^{2} \cdot$ Véronique Leblond $^{7} \cdot$ Khê Hoang-Xuan $^{1} \cdot$ Mehdi Touat $^{1}$}

1 Service de Neurologie 2-Mazarin, Sorbonne Université, Inserm, CNRS, UMR S 1127, Institut du Cerveau Et de La Moelle Épinière, ICM, AP-HP, Hôpitaux Universitaires La Pitié Salpêtrière - Charles Foix, 47-83 boulevard de l'Hôpital, 75013 Paris, France

2 Service de Neurologie, Université de Paris, AP-HP, Hôpital Saint Louis, 75010 Paris, France

3 Laboratoire de virologie, AP-HP, Hôpitaux Universitaires La Pitié Salpêtrière - Charles Foix, 75013 Paris, France

4 Sorbonne Université, INSERM 1136, Institut Pierre Louis D’Epidémiologie Et de Santé Publique, 75013 Paris, France

5 Service de Maladies infectieuses et Tropicales, AP-HP, Hôpitaux Universitaires La Pitié Salpêtrière - Charles Foix, 75013 Paris, France

6 Département d'anesthésie et réanimation, AP-HP, Hôpitaux Universitaires La Pitié Salpêtrière - Charles Foix, Groupe Hospitalier Pitié-Salpêtrière, 75013 Paris, France
7 Service d'hématologie clinique, AP-HP, Hôpitaux Universitaires La Pitié Salpêtrière - Charles Foix, Groupe Hospitalier Pitié-Salpêtrière, 75013 Paris, France

8 Service d'Hématologie, Université de Paris, AP-HP, Hôpital Saint Louis, 75010 Paris, France

9 Service de neurochirurgie, AP-HP, Hôpitaux Universitaires La Pitié Salpêtrière - Charles Foix, Groupe Hospitalier Pitié-Salpêtrière, 75013 Paris, France

10 Service de Neuropathologie Laboratoire Escourolle, Sorbonne Université, Inserm, CNRS, UMR S 1127, Institut du Cerveau Et de La Moelle Épinière, ICM, AP-HP, Hôpitaux Universitaires La Pitié Salpêtrière - Charles Foix, 75013 Paris, France

11 Service de neuroradiologie, AP-HP, Hôpitaux Universitaires La Pitié Salpêtrière - Charles Foix, 75013 Paris, France 